\title{
Fabrication and Properties of Amorphous Zinc Oxynitride Thin Films
}

\author{
J. Kaczmarski*, M.A. Borysiewicz, K. Pągowska and E. Kamińska \\ Institute of Electron Technology, al. Lotników 32/46, 02-668 Warsaw, Poland
}

(Received October 20, 2015; in final form December 3, 2015)

\begin{abstract}
$\mathrm{Zn}-\mathrm{O}-\mathrm{N}$ thin films fabricated by reactive radio frequency magnetron sputtering have been investigated for their compositional, structural, transport and optical properties. In contrast to processes in which the reaction for either the oxide or the nitride is dominant, the multireaction process yields a substantially amorphous films with the Hall mobility within the range from 15 to $80 \mathrm{~cm}^{2} /(\mathrm{V} \mathrm{s})$. In addition, it has been observed that the Hall mobility increases for $\mathrm{Zn}-\mathrm{O}-\mathrm{N}$. Since it has a narrower bandgap than $\mathrm{ZnO}$, it is put forward that the high mobility is due to the valence band maximum in this material lying above the trap states in the gap commonly observable in $\mathrm{ZnO}$. These traps originate from oxygen vacancies and are localized at the bottom of the band gap influencing the carrier mobility.
\end{abstract}

DOI: 10.12693/APhysPolA.129.150

PACS: 81.05.Gc, 81.15.Cd, 72.80.Ng, 73.61.Jc

\section{Introduction}

Multi-cationic and multi-anionic amorphous oxide semiconductors (AOS) are the subject of intensive theoretical and experimental research aimed at applications in thin-film transistors (TFTs), as an enabling technology for novel displays, communication, computing, sensing, and identification devices $[1,2]$. This material group offers a wide range of potential applications - from transparent semiconductor devices and electronic systems, to a whole range of optical and chemical sensors, manufactured on both rigid and flexible substrates. Oxide semiconductors composed of $\mathrm{In}, \mathrm{Ga}, \mathrm{Sn}, \mathrm{Ag}$, and $\mathrm{Zn}$ cations combine an X-ray amorphous microstructure and optical transparency in visible wavelength spectrum with controllable electrical conductivity from highly conductive to highly-resistive. An unique aspect of AOS is that the carrier mobility is not sensitive to the thin film microstructure, as is case of conventional covalently-bonded semiconductors. This fact arises from the nature of the chemical bonding in these metal oxides. Carrier transport in covalently-bonded materials, such as $\mathrm{Si}$, is carried out primarily through anisotropic $s p^{3}$ orbitals, so that introducing randomness into the structure greatly reduces bond overlap and carrier mobility. In AOS, the higher ionicity of the bonds leads to the formation of the conduction band from the spherical $s$ orbitals. Since the overlap of $s$ orbitals is not significantly altered by the introduction of structure randomness, carrier transport and thus mobility is insensitive to the amorphization [3].

Amorphous In-Ga-Zn-O (a-IGZO) has attracted much attention as a channel material for TFTs owing to its electron mobility above $10 \mathrm{~cm}^{2} /(\mathrm{V} \mathrm{s})$ already achieved by room-temperature fabrication methods [1]. However,

*corresponding author; e-mail: kaczmarski@ite.waw.pl a mobility considerably higher than $10 \mathrm{~cm}^{2} /(\mathrm{V} \mathrm{s})$ cannot be achieved in such multi-metal compounds due to potential fluctuations within the conduction band, that arise from the random distribution of the different cations. The presence of potential barriers may be explained based on the model of overlapping of $s$-orbitals wave functions. Even by excluding the complexity of randomness in an amorphous phase, it may not be thermodynamically favorable to pack cations of different metals in such a way that the distance between neighboring metal cations will be smaller than the distance of the interactions between $s$-orbitals of these cations $[3,4]$. Therefore, it could be expected that there will be areas where the inter-cation distances are larger than the radii of their $s$-orbitals, causing interruptions in the conduction band minimum, giving rise to potential barriers, and limiting carrier mobility. Another factor suppressing carrier mobility is the presence of donor-like oxygen vacancies $\left(\mathrm{V}_{o}\right)$ located within the wide bandgap of AOS. Therefore, a promising approach to further increase carrier mobility in AOS is the formation of a single-metal compound with multiple anions suppressing such potential fluctuations $[2,5]$.

Several research groups already reported the possibility to control the valence band through substituting oxygen anions by non-oxide anions, such as $\mathrm{N}^{3-}, \mathrm{S}^{2-}$, $\mathrm{Se}^{2-}, \mathrm{F}^{-}[6-8]$.

Here, we present the results of the incorporation of larger size anions, namely $\mathrm{N}^{3-}$ with higher $p$ orbital energy than oxygen into $\mathrm{ZnO}$. At large nitrogen densities, such approach does not cause $p$-type doping but results in the formation of an amorphous alloy, where the anion site disorder stabilizes the amorphous phase, forming amorphous $\mathrm{Zn}-\mathrm{O}-\mathrm{N}$ thin films. This does not lower the electron mobility because the disorder is in valence band states, and not the conduction band states. Another important effect is that the $\mathrm{N} 2 p$ orbital lies higher than the O $2 p$ orbital, so that the new $\mathrm{N} 2 p$ states raise the valence band maxima above its energy in $\mathrm{ZnO}[5,6]$. As a result, 
CBM
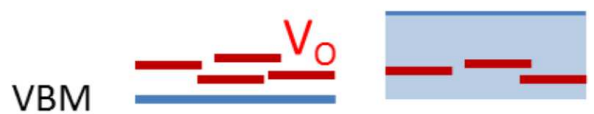

\section{Valence band}

Fig. 1. Schematic band diagrams of $\mathrm{ZnO}, \mathrm{Zn}-\mathrm{O}-\mathrm{N}$, and $\mathrm{Zn}_{3} \mathrm{~N}_{2}$, showing valence band maximum (VBM), conduction band minimum (CBM) and donor-like states within the bandgap originated from oxygen vacancies $\left(\mathrm{V}_{o}\right.$ - red lines).

the initial localized trap states are now covered up by the higher valence band maximum states, so they can no longer give lower the carrier mobility [2]. A schematic of this idea is depicted in Fig. 1.

\section{Experimental details}

$\mathrm{Zn}-\mathrm{O}-\mathrm{N}$ thin films with a thickness of $100 \mathrm{~nm}$ were deposited on unheated quartz substrates, by a reactive radio frequency (rf) magnetron sputtering in $\mathrm{Ar} / \mathrm{N}_{2} / \mathrm{O}_{2}$ plasma in a Leybold Z400 system, from $75 \mathrm{~mm}$ diameter metallic $\mathrm{Zn}$ target of $4 \mathrm{~N}$ purity, at a pressure $\left(P_{\text {tot }}\right)$ of $1 \mathrm{~Pa}$. The oxygen to nitrogen ratio $\left(\mathrm{O}_{2}: \mathrm{N}_{2}\right)$ in the sputtering atmosphere was varied between 1:20 and 1:100. The cathode current was maintained at the level of $150 \mathrm{~mA}$. The composition of $\mathrm{ZnON}$ films was inferred from the Rutherford backscattering spectrometry (RBS) using $2 \mathrm{MeV} \mathrm{He}^{+}$. To enable precise measurements of composition, complementary Zn-O-N films were deposited on (111)-oriented Si substrate. SIMNRA program was used to simulate experimental RBS spectra. Structural analysis was performed using Philips X'Pert Pro Alpha1 Multi Purpose Diffractometer. The resistivity of thin films was determined from four-point probe measurements with a JANDEL RM3-AR apparatus. Carrier type, concentration and mobility were evaluated from the Hall measurements in the van der Pauw configuration at a magnetic field of $0.44 \mathrm{~T}$, by means of a Phys Tech RH2035 setup. Ohmic contacts were fabricated by sputter deposition of $\mathrm{Ti} / \mathrm{Al}$ bilayer. For optical characterization, transmittance measurements in the wavelengths range $250-950 \mathrm{~nm}$, by means of a SENTECH SE800E spectroscopic ellipsometer were performed.

\section{Results and discussion}

By varying the $\mathrm{N}_{2}$ and $\mathrm{O}_{2}$ content in plasma at fixed Ar flow rate, the anion ratio in $\mathrm{Zn}-\mathrm{O}-\mathrm{N}$ was modulated, while maintaining $\mathrm{Zn}$ concentration at $\approx 55$ at.\%. As presented in Fig. 2a, the nitrogen content was found to increase with the $\mathrm{O}_{2}: \mathrm{N}_{2}$ ratio, constituting from below 5 to $42 \%$ of the atoms in the film, while the oxygen fraction decreased from $40 \%$ to $3 \%$. Figure $2 \mathrm{~b}$ shows the $\mathrm{XRD}$ measurement results of the $\mathrm{Zn}-\mathrm{O}-\mathrm{N}$ films. Wideangle XRD patterns for all samples show only one broad
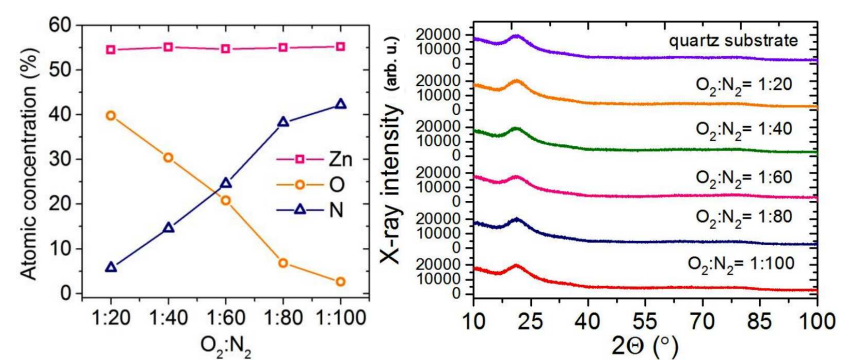

Fig. 2. Atomic composition (a) and diffraction patterns (b) of $\mathrm{Zn}-\mathrm{O}-\mathrm{N}$ films as a function of $\mathrm{O}_{2}: \mathrm{N}_{2}$ ratio.

peak at $2 \Theta=22^{\circ}$ corresponding to the quartz substrate, implying that all films have an amorphous or nanocrystalline microstructure.

Figure 3 a shows resistivity and carrier concentration of the $\mathrm{Zn}-\mathrm{O}-\mathrm{N}$ films as a function of the oxygen to nitrogen ratio in the deposition atmosphere. All fabricated films were $n$-type semiconductors when characterized by the Hall measurements. The carrier concentration significantly increases from $1.2 \times 10^{16}$ to as $3.4 \times 10^{18} \mathrm{~cm}^{-3}$ as nitrogen content in sputtering plasma increases, indicating that oxygen may act as a carrier suppressor. The Hall mobility increases as the carrier concentration increases, which might be explained by the percolation model similar to the case of a-IGZO, and the Hall mobility above $80 \mathrm{~cm}^{2} /(\mathrm{V} \mathrm{s})$ was obtained at carrier concentrations equal to $n=3.4 \times 10^{18} \mathrm{~cm}^{-3}$ (see Fig. $3 \mathrm{~b}$ ).
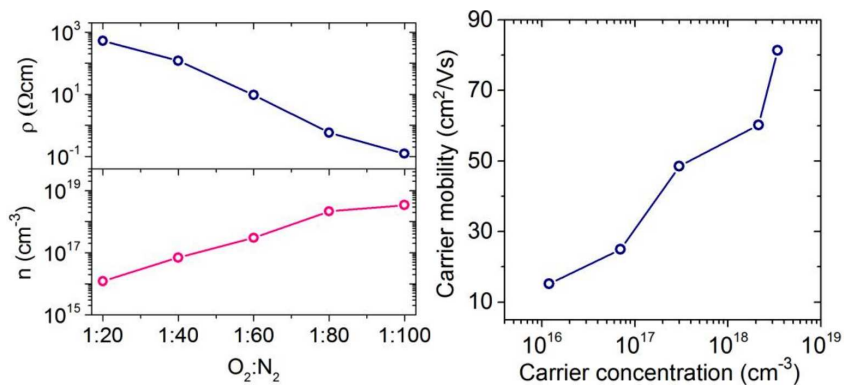

Fig. 3. Carrier concentration and resistivity of a-IGZO as a function of percentage of oxygen (a) and dependence of Hall mobility on the carrier concentration in a-IGZO (b).

It is noted that the Hall mobility of $\mathrm{Zn}-\mathrm{O}-\mathrm{N}$ increases with the carrier concentration more rapidly than for aIGZO. This could be attributed to the relatively low potential barriers above the conduction band edge of $\mathrm{Zn}-$ $\mathrm{O}-\mathrm{N}$ that is composed of single $\mathrm{Zn} 4 s$ orbitals, unlike the random distribution of multiple cations ( $\mathrm{In}, \mathrm{Ga}, \mathrm{Zn}$ ) in a-IGZO.

The results of transmission measurements for different $\mathrm{O}_{2}: \mathrm{N}_{2}$ ratios are presented in Fig. 4a. It can be seen that a lower nitrogen content in the sputtering atmosphere yields higher transparency for the films in accordance with the formation of $\mathrm{ZnO}$. The spectral characteristic curves show the enhanced absorption of light in 

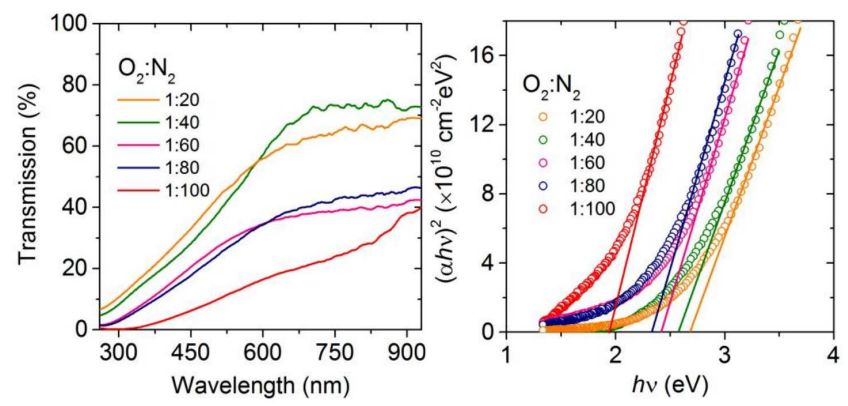

Fig. 4. Optical transmission (a) and Tauc plot (b) of $\mathrm{Zn}-\mathrm{O}-\mathrm{N}$ films deposited at various $\mathrm{O}_{2}: \mathrm{N}_{2}$ ratio.

the visible wavelength range. Optical bandgaps $\left(E_{\text {opt }}\right)$ of fabricated materials were estimated from the Tauc model $\left(\alpha h \nu \propto\left(h \nu-E_{\text {opt }}\right)^{n}\right.$, where $\alpha$ is absorption coefficient, $h \nu$ is the photon energy, and $n=2$ for allowed indirect transition) by extrapolation of linear Tauc's region to $(\alpha h \nu)^{2}=0$ [9]. Indeed, the evaluated optical bandgaps, presented in Fig. 4b, lie within the bandgap range of $\mathrm{ZnO}$ $\left(E_{\text {opt }}=3.2 \mathrm{eV}\right)$ and $\mathrm{Zn}_{3} \mathrm{~N}_{2}\left(E_{\text {opt }}=1.1 \mathrm{eV}\right)$, and monotonically decreases from $E_{\text {opt }}=2.73 \mathrm{eV}$ for $\mathrm{Zn}_{54} \mathrm{O}_{40} \mathrm{~N}_{6}$ to $E_{\text {opt }}=1.92 \mathrm{eV}$ for $\mathrm{Zn}_{55} \mathrm{O}_{3} \mathrm{~N}_{42}$. It is also noted that with increase of nitrogen content the Urbach tail decreases. Since the Urbach tail corresponds to the density subgap states, it suggests that the optical bandgap is narrowed as a consequence of elevation of valence band maximum above localized trap states (see Fig. 1), properly caused by the formation of $\mathrm{Zn}-\mathrm{N}$ bonds which have smaller ionicity than $\mathrm{Zn}-\mathrm{O}$ bonds $[2,5,6]$.

\section{Conclusions}

Amorphous $\mathrm{Zn}-\mathrm{O}-\mathrm{N}$ thin films were fabricated by reactive $\mathrm{rf}$ magnetron sputtering and characterized by use of complementary methods of compositional and microstructural analysis. The oxygen to nitrogen ratio in the deposition atmosphere significantly affects the electrical properties of the films. In contrast to processes in which the reaction for either the oxide or the nitride is dominant, the multireaction process yields a substantially amorphous films with the Hall mobility within the range from 15 to $80 \mathrm{~cm}^{2} /(\mathrm{V} \mathrm{s})$. The optical bandgap $\mathrm{Zn}-\mathrm{O}-\mathrm{N}$ is in the range of $1.92 \mathrm{eV}$ to $2.73 \mathrm{eV}$. We attribute the enhancement of transport properties to the narrowing of $E_{\text {opt }}$ by elevation of valence band maximum over native trap states localized in the lower band gap region. Chemical, transport and optical properties make $\mathrm{Zn}-\mathrm{O}-\mathrm{N}$ technology strongly promising to achieve high mobility required the realization of high-performance thin film transistors.

\section{Acknowledgments}

This study was partially supported by the European Union within European Regional Development Fund, through grant Innovative Economy (POIG.01.03.01-00159/08, "InTechFun").

\section{References}

[1] A. Nathan, S. Lee, S. Jeon, J. Robertson, J. Display Technol. 10, 917 (2014).

[2] H.-S. Kim, S.H. Jeon, J.S. Park, T.S. Kim, K.S. Son, J.-B. Seon, S.-J. Seo, S.-J. Kim, E. Lee, J.G. Chung, H. Lee, S. Han, M. Ryu, S.Y. Lee, K. Kim, Nature Sci. Rep. 3, 1459 (2013)

[3] K. Nomura, H. Ohta, A. Takagi, T. Kamiya, M. Hirano, H. Hosono, Nature 432, 488 (2004).

[4] T. Kamiya, H. Hosono, ECS Trans. 54, 103 (2013).

[5] Y. Ye, R. Lim, J.M. White, J. Appl. Phys. 106, 074512 (2009).

[6] E. Lee, A. Benayad, T. Shin, H. Lee, D.-S. Ko, T.S. Kim, K.S. Son, M. Ryu, S. Jeon, G.-S. Park, Nature Sci. Rep. 4, 4948 (2014).

[7] E. Premalal, N. Dematage, S. Kaneko, A. Konno, Thin Solid Films 520, 6813 (2014).

[8] T. Umebayashi, T. Yamaki, H. Itoh, K. Asai, Appl. Phys. Lett. 81, 454 (2002).

[9] J. Tauc, Phys. Today 29, 23 (1976). 